\title{
Genome-wide expression profiling reveals distinct clusters of transcriptional regulation during bovine preimplantation development in vivo
}

\author{
W. A. Kues a,1, S. Sudheerb,1, D. Herrmanna, J. W. Carnwath ${ }^{a}$, V. Havlicek ${ }^{\text {b }}$, U. Besenfelder ${ }^{\text {, }}$, H. Lehrachc, J. Adjayec,
} and H. Niemann ${ }^{\mathrm{a}, 2}$

anstitute of Farm Animal Genetics, Friedrich Loeffler Institute, Mariensee, 31535 Neustadt, Germany; 'Reproduction Centre-Wieselburg, University of Veterinary Medicine, 1210 Vienna, Austria; and bepartment of Vertebrate Genomics, Max Planck Institute for Molecular Genetics, 14195 Berlin, Germany

Edited by Neal L. First, Mississippi State University, Mississippi State, MS, and approved October 16, 2008 (received for review June 12, 2008)

Bovine embryos can be generated by in vitro fertilization or somatic nuclear transfer; however, these differ from their in vivo counterparts in many aspects and exhibit a higher proportion of developmental abnormalities. Here, we determined for the first time the transcriptomes of bovine metaphase II oocytes and all stages of preimplantation embryos developing in vivo up to the blastocyst using the Affymetrix GeneChip Bovine Genome Array which examines approximately 23,000 transcripts. The data show that bovine oocytes and embryos transcribed a significantly higher number of genes than somatic cells. Several hundred genes were transcribed well before the 8-cell stage, at which the major activation of the bovine genome expression occurs. Importantly, stage-specific expression patterns in 2-cell, 4-cell, and 8-cell stages, and in morulae and blastocysts, were detected, indicating dynamic changes in the embryonic transcriptome and in groups of transiently active genes. Pathway analysis revealed $>120$ biochemical pathways that are operative in early preimplantation bovine development. Significant differences were observed between the mRNA expression profiles of in vivo and in vitro matured oocytes, highlighting the need to include in vivo derived oocytes/embryos in studies evaluating assisted reproductive techniques. This study provides the first comprehensive analysis of gene expression and transcriptome dynamics of in vivo developing bovine embryos and will serve as a basis for improving assisted reproductive technology.

embryo | transcriptomics | assisted reproductive techniques |

gene clusters | embryonic genome activation

돈 arly mammalian embryogenesis critically depends on a tightly controlled, well orchestrated program of gene expression. Fertilization and the early cleavage stages are associated with massive epigenetic reprogramming of the paternal and maternal chromatin and initiated by maternal RNA and proteins accumulated during oogenesis and the final stages of oocyte maturation (1). Large scale synthesis of messenger RNA from the diploid embryonic genome is initiated at a species-specific time point. This occurs in murine embryos at the end of the first cell cycle, in human embryos at the 4-cell stage and in bovine embryos at the 8-cell stage (2-4). The characterization of normal maternal and embryonic transcriptomes is essential for understanding embryogenesis and for improving of assisted reproductive techniques (ARTs).

Transcriptomic studies of in vivo developing embryos recovered from livestock species such as cattle are valuable for two main reasons $(5,6)$. First, Bos taurus is an economically important species for which advanced reproduction technology has been developed (7). The use of in vivo developing embryos establishes a standard against which embryos derived by various in vitro techniques can be compared. This will help in the analysis of mechanisms underlying embryonic losses and for improving in vitro culture systems. Second, the bovine embryo is increasingly used as an alternative to the mouse for the analysis of mammalian preimplantation development and the data are critical for understanding the causes of aberrations in embryonic and fetal development in humans (8-14). Due to ethical considerations, research on human embryos is restricted by law in most countries, and where it is permitted, only surplus (often poor quality) in vitro produced embryos can be used. Unfortunately, in vitro culture is known to affect the morphological and biochemical characteristics of mammalian embryos in a significant manner $(15,16)$.

Here, we report on the transcriptome dynamics of preimplantation bovine embryos, developing in their natural environment and provide the first comprehensive data set representing preimplantation development in vivo.

\section{Results}

Global Data Analysis. Using improved RNA extraction and amplification techniques, we found highly similar profiles between replicates of the same developmental stage (Pearson's correlation coefficients ranging from 0.911 to 0.998 ), reflecting the high reproducibility of the procedure. The Pearson correlation coefficients between the profiles of consecutive embryonic stages reflect the degree of change in transcriptomes (Table S1). In total, 12,000 to 14,500 transcripts were detected in each in vivo stage (Table 1).

Stage Specific Dynamics in Transcription. The greatest difference was found between early bovine preimplantation development (oocyte to 4-cell stage) and later stages of development (8-cell to blastocyst) (Fig. 1A), reflecting the transition from maternal genome to embryonic genome transcription. The highest number of differentially regulated genes was found between 4-cell and 8-cell stage (Fig. $1 A$ and $B$ ), demonstrating the major onset of embryonic genome activation (EGA) at the 8 -cell stage $(3,4)$. Amongst the several hundred transcripts identified, genes previously known to be embryonically expressed include $D N M T 3 A$, claudin $(C L D N)$, tight junction protein $3(Z O 3)$, and p53 tumor suppressor phosphoprotein (TP53) $(17,18)$.

The fact that complex changes were observed in the transcriptome during the first cleavage stages indicates that many transcriptional adjustments take place before major EGA ( $p$-call

Author contributions: J.W.C., H.L., J.A., and H.N. designed research; D.H., V.H., and U.B. performed research; W.A.K., S.S., and D.H. analyzed data; and W.A.K., S.S., J.W.C., J.A., and H.N. wrote the paper.

The authors declare no conflict of interest.

This article is a PNAS Direct Submission.

Data deposition: The data reported in this paper have been deposited in the Gene Expression Omnibus (GEO) database, www.ncbi.nlm.nih.gov/geo (accession no. GSE12327). ${ }^{1}$ W.A.K. and S.S. contributed equally to this work.

2To whom correspondence should be addressed. Email: heiner.niemann@fli.bund.de.

This article contains supporting information online at www.pnas.org/cgi/content/full/ 0805616105/DCSupplemental.

C 2008 by The National Academy of Sciences of the USA 
Table 1. Detected transcripts in in vivo stages

\begin{tabular}{lc} 
Stage & No. of transcripts \\
\hline Metaphase II oocyte & 12,049 \\
Zygote & 12,187 \\
2-cell stage & 14,428 \\
4-cell stage & 14,032 \\
8-cell stage & 13,025 \\
Morula & 12,576 \\
Blastocyst & 12,956 \\
\hline
\end{tabular}

values; Fig. 1C, Fig. S1). In our dataset, 2,473 genes were significantly up-regulated (at least 2-fold) in 8-cell embryos, morulae, and blastocysts, compared to 2-cell and/or 4-cell stages. These can be classified as genes involved in EGA. Altogether 321 genes $(13 \%)$ were up-regulated in the 2-cell stage and 285 genes $(11.5 \%)$ were up-regulated in the 4-cell stage compared to zygotes; 197 (8\%) genes were common to both of these two sets and remained up-regulated until the blastocyst stage.

A group of 48 genes were up-regulated in the 2-cell stage, down regulated in the 4-cell stage, and then up-regulated again remaining highly transcribed to the blastocyst stage. Among these were IL18 and tumor protein, translationally-controlled 1 (TPT1); the latter is one of the most abundantly expressed genes in both human and bovine blastocysts and its role in the initiation of embryonic genome activation seems to be evolutionarily conserved (18).

Another interesting pattern was seen in 22 genes (Fig. S1, Table S1) for which transcript levels peaked at the 2-cell stage. These 2-cell-specific genes, along with the 48 genes mentioned above, make a set of 70 genes, which may be important factors for the onset of embryonic genome activation. Of these 70 genes, 49 were previously unknown. The known genes include cysteinerich, angiogenic inducer, 61 (CYR61) which is an important determinant of the genetic reprogramming that occurs in mechanically challenged cells (19) and carboxypeptidase E (CPE), which is involved in the biosynthesis of peptide hormones and neurotransmitters known to activate mitogen-activated protein kinase (20).

In total, approximately 350 significantly expressed genes could be identified which are transcribed in a stage-specific pattern before the major onset of EGA which occurs at the 8-cell stage. This implies specific functions of these genes during early cleavage stages.

Confirmation of Array Data by Real Time RT-PCR. Two independent RT-PCR analyses of the amplified RNA (aRNA) verified that the array data accurately reflect the relative abundance of selected amplified transcripts in the samples (Fig. S2).

To obtain independent verification, individual embryos were collected at each stage and analyzed by real time PCR. These were compared with published expression profiles of genes, known to be important for mouse, human, and bovine development (Fig. S3). BMP15, KIT, GDF9, STAT3, C-MOS and ZP2, 3, 4 are oocyte markers $(2,18,21-25)$. The array analysis of bovine embryos revealed expression of $Z P 2,3,4, G D F 9, B M P 15$, cadherin (CDH3), but also OCT4 (POU5F1), CLDN, ZO3, and TP53 (Fig. S3a).

The maternal expression profiles of $D N M T 1, Z P 2$, and $G D F 9$ and the embryonic expression profiles of GLUT8, DNMT3A and $V D A C 2$ were confirmed by real time RT-PCR from single embryos $(n=6)$. Genes with stage-specific expression (SPP1, $B M P R I B, S U B T 4 H 1)$ or with expression before EGA (POLR1C, $C T S H, C D 48)$ by the Affymetrix array analysis, were checked by real time RT-PCR (Fig. 2).

In control experiments for the RT-PCR, reverse transcription of oocyte RNAs was primed with either poly(T) oligos or random hexamers. Identical results were obtained irrespective of the primers used, indicating that the modifications of the Poly (A) tails in oocytes did not affect amplification and hybridization under our experimental conditions (data not shown).

Cluster Profiles of Transcriptomes. Clusters with similar expression profiles were grouped for a global view of preimplantation transcriptome dynamics (Fig. 3, Table S2). The 4,173 maternal transcripts could be grouped as high, medium, or rare transcript abundance in oocytes. These maternal transcript clusters were
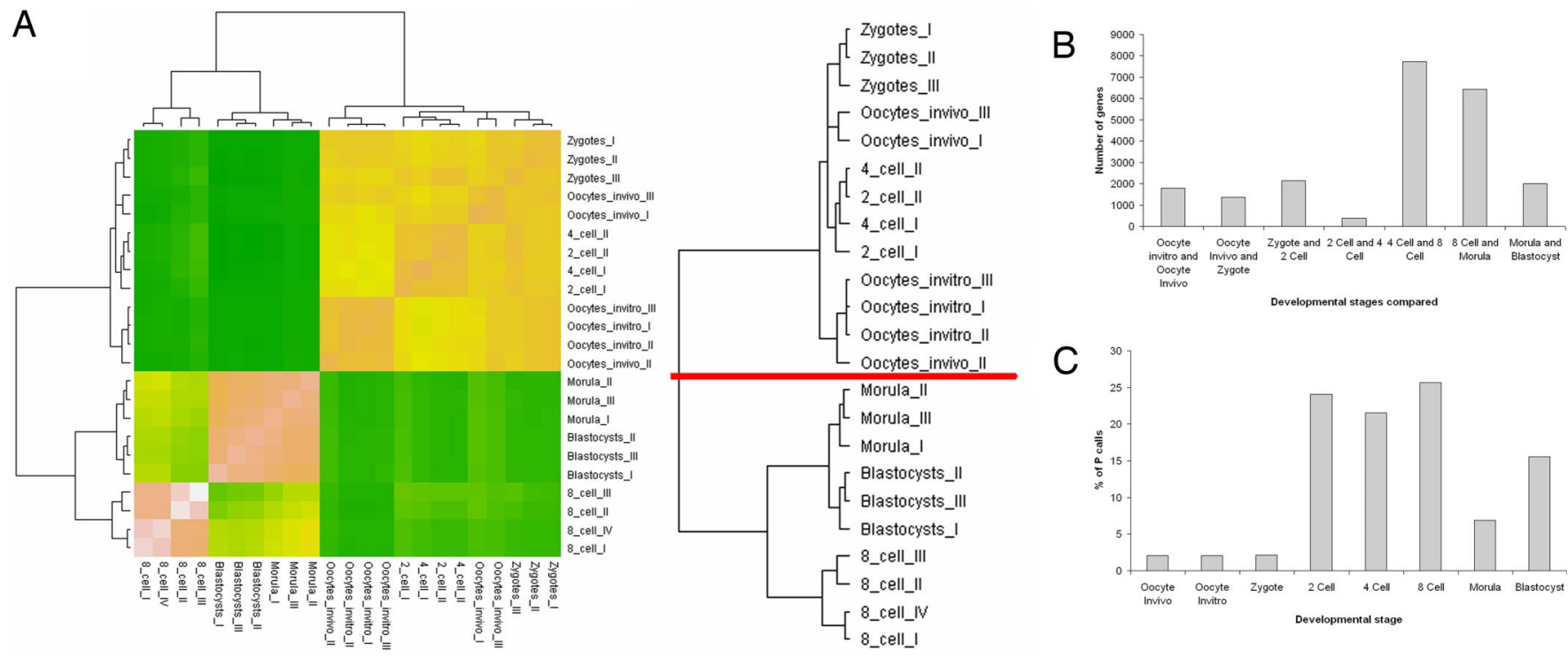

Fig. 1. Global analysis of transcriptome features in naturally developing bovine embryos. $(A)$ shows Pearson correlation coefficients between replicates from all of the bovine embryonic stages as a "heat map" in which the color spectrum ranging from orange through yellow to green represents Pearson Correlation Coefficient values ranging from 0.519 to 1 , and hierarchial clustering of various developmental stages. $(B)$ Number of differentially regulated genes ( $>2$-fold) of subsequent stages. (C) Stage-exclusive expressed genes ( $P$ call percentages). 

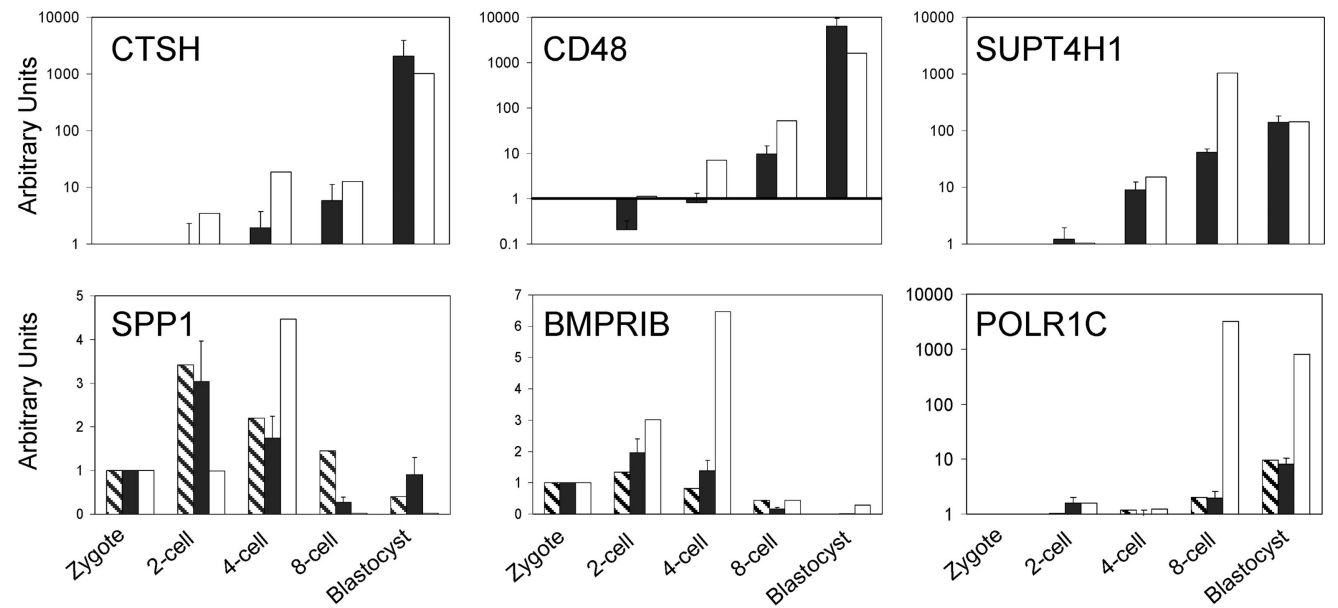

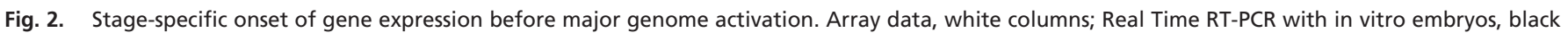
columns; Real Time RTPCR with in vivo derived embryos, shaded columns.

characterized by a drop of transcription levels between the 4- and 8-cell stages. A second group of genes showing embryonic transcription includes three clusters representing 3,505 genes (Fig. 3). Significantly increased transcript levels were found from the 4-cell to 8-cell stage onwards, indicating genes (ID2, ZO3, CLDN4, TP53, etc.) transcribed during major embryonic activation.

In addition, stage-specific clusters were detected. The clusters of 8-cell enriched transcripts and reduced transcript prevalence in 8-cell stage are depicted (Fig. $3 C$ ). The stage-specific clusters of zygotes, 2-4-cell embryos, morulae, and blastocysts, are given in. Table S2.

A novel finding is the rapid degradation of maternal messenger RNAs that occurs after the 4-cell stage concomitantly with the major onset of embryonic transcription at the 8-cell stage

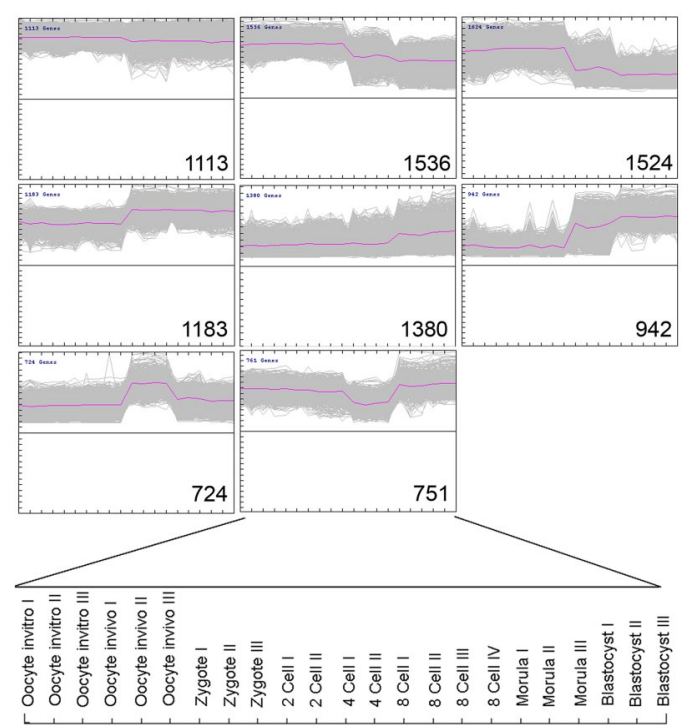

Fig. 3. Identification of new expression profiles by cluster analysis (grouping of genes by various algorithms (f.ex. K-means) according to their similarity in expression profiles across a set of experiments or samples). The expression graphs were generated by the K-Means clustering algorithm. (Top row) Three clusters showing maternal expression profiles and high, medium, or low expression at zygote stage. (Middle row) Three clusters with embryonic expression profiles and high, medium, or low expression at the blastocyst stage. (Bottom row) Two clusters with 8-cell-specific up and down-regulated transcript levels. Embryonic stages are depicted on the $x$ axis.
(Fig. $3 A$ ), instead of the previously assumed gradual degradation of maternal messenger RNAs $(26,27)$. This indicates that maternal RNA is actively degraded in a coordinated manner and that degradation and genome activation are tightly linked.

Oocyte Enriched Genes. A set of 429 genes were found to be at least 2 -fold significantly regulated in in vivo oocytes, with respect to all other stages. Of this set, 35 genes were up-regulated only in oocytes (for example: PGF, RAD52, CST6, CPA1, POLR2J2). Some of these genes are depicted in Fig. $4 A$, which shows a decreasing expression pattern during preimplantation development (Table S3).
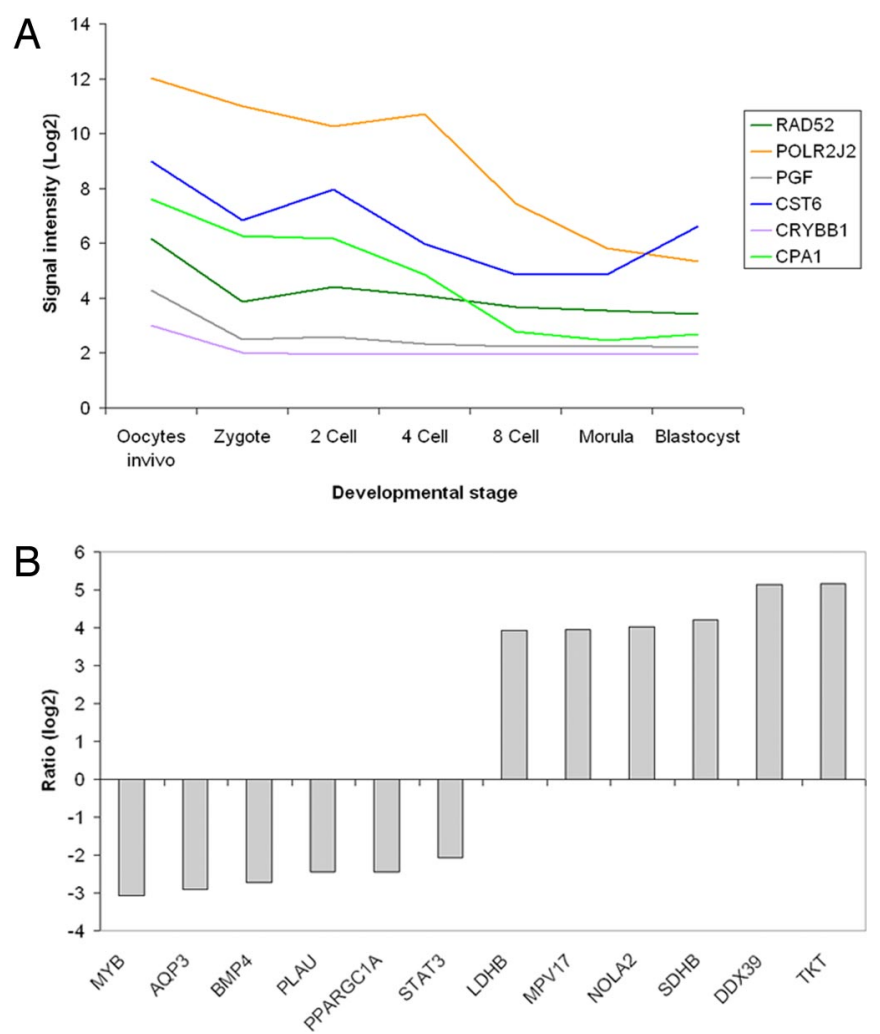

Fig. 4. Identification of oocyte enriched genes and of genes differentially expressed between in vivo and in vitro produced embryos. (A) Oocyte enriched genes. $(B)$ Genes differentially expressed between in vivo and in vitro oocytes. 
Comparison of the Transcriptomic Profile in Vivo and in Vitro Matured Bovine Oocytes. In in vivo derived oocytes, more genes were detected than their in vitro derived counterparts (12,049 vs. 11,332 transcripts) and 1,799 transcripts were significantly differentially regulated by 2 -fold between the two types of oocytes. Of these, the majority of the genes $(1,267)$ were up-regulated in in vivo derived oocytes compared to in vitro cultured oocytes (532). For example, NOLA2, SDHB, TKT, DDX39 and MPV17 were up-regulated, while $M Y B, A Q P 3, O R P 150, B M P 4$ and $S T A T 3$ were less abundant in in vivo derived oocytes than in their in vitro matured counterparts (Fig. $4 B$ ). This study gave similar results to a previously published Affymetrix bovine chip analysis which identified 821 transcripts that were differentially expressed between in vivo and in vitro matured bovine oocytes (28).

Pathways Operative during Preimplantation Development. Pathway analysis delineated essential signaling and metabolic pathways in embryonic development. MAPK, TGF-beta, insulin signaling, and metabolic processes such as glycolysis and oxidative phosphorylation were functional and components of these pathways were subject to significant changes in transcript levels during preimplantation development (Fig. S4). It is noteworthy that the most prominent differences occurred between the 4-cell and 8 -cell stages, reflecting an early transition from maternal to embryonic gene expression.

\section{Discussion}

Here, we provide the transcriptomes of in vivo grown oocytes and early embryonic stages from the bovine species (Bos taurus). These data are an important step toward an unbiased view of gene transcription and regulation during early mammalian development. Most studies on embryonic transcriptomes have been focused on the mouse (29-32), which shows peculiarities of preimplantation development potentially limiting its utility as a model for human embryogenesis. Previous studies have used bovine embryos from in vitro sources and/or smaller arrays, which yield fragmentary pictures of the transcriptional activity in early embryos (for review see ref. 33).

In this study, special care was taken to obtain the natural transcriptome profile. Advanced ultrasound guided follicular aspiration and laparoscopic techniques were used to isolate oocytes and oviductal embryonic stages with minimal invasiveness from a selected group of fertile female cattle. Embryos were frozen immediately after isolation from the reproductive tract to preserve the in vivo transcriptome as best as possible. The presence of $>12,000$ transcripts in each embryonic stage is significantly higher than the 8,000 transcribed genes typical of several somatic tissues from humans or mouse (32) and supports the notion that embryonic cells express a greater proportion of the genome than differentiated somatic cells, reflecting the uncommitted status of the pluripotent blastomeres. This dataset of expressed gene and transcription profiles can be used to assess the influence of culture conditions and handling in vitro. Bovine and human preimplantation development are similar with respect to the timing of epigenetic reprogramming and activation of embryonic genome activation and the results of this study may be valuable for the evaluation of human embryos produced by various ARTs.

Several hundred genes are transcribed before the major onset of gene expression. Their low levels of expression may explain why their presence was not detected in previous, less sensitive analyses. Previous reports have alluded to minor bovine embryonic genome activation between the zygote and 4-cell stage (34-37). Our data show that this minor EGA occurs between the 2-4-cell stages. Among the genes which were up-regulated in 2-4-cell stages, seven transcripts ( $S A R S$, IL18, CRABP1, ACO2, TXN2, SLC38A2, SLC25A3) were previously identified as enriched in normal 8-cell bovine embryos relative to alpha-amanitin-treated 8-cell embryos (4).

An important novel finding of this study was the discovery of several genes expressed exclusively at only one stage of preimplantation development. The transient stage-exclusive expression suggests that these genes are critical only for specific stages of development. Stage-exclusive expression of the eukaryotic translation initiation factor eIF-4c has been reported in mouse embryos at the 2-cell stage, when murine embryonic genome activation occurs (38). The bovine orthologous genes of eukaryotic translation initiation factor, EIF2, EIF3, EIF4 and EIF5, become selectively up-regulated at the 8-cell stage, when the bovine embryonic genome is activated indicating a conserved role of this gene family in murine and bovine embryos.

Transcripts related to DNA methylation, histone modification, and chromatin remodeling could be clustered based on maximal or minimal expression at the 8-cell stage, reflecting critical molecular remodeling at this time point. These epigenetic modifications are key regulators of developmental processes during preimplantation development $(39,40)$ and spurious epigenetic marks may have long-lasting consequences for the offspring. The identification of unique expression profiles for these groups of genes in embryos grown in utero makes it feasible to investigate how in vitro culture conditions affect the expression of these critical remodelers of the embryonic genome and subsequently to optimize in vitro culture conditions.

The limitations of the data set presented in this study are $(i)$ gene ontology information for the probe sets (each gene is represented as 11-22 probes) of the bovine Affymetrix chip is still incomplete and probes for some known developmentally important genes, such as telomerase (TERT) are lacking, (ii) the in vivo derived embryos were collected from superovulated donor cows because cleavage and blastocyst stage embryo collection from non-superovulated donor cows is not feasible. The viability of bovine blastocysts derived from superovulated donors is well established $(41,42)$ and forms the basis for the global embryo transfer industry with approximately 670,000 in vivo and approximately 290,000 in vitro derived bovine embryos transferred in 2006 (43).

In conclusion, this is the first comprehensive evaluation of transcriptome dynamics during bovine preimplantation development in vivo, it represents the natural physiological status as accurately as possible because oocytes and embryos were directly isolated from the reproductive tract of live donors, frozen, and processed for analysis. Results indicate a highly dynamic transcriptome both before and after the major embryonic genome activation and reveal the coordinated breakdown of maternal transcripts coinciding with major genome activation. The early expression of approximately 350 genes may explain the sensitivity of embryos to changes in their environment. These data can be used to assess the impact of various assisted reproductive techniques on the embryo and improve the safety of these techniques in both bovine and human reproduction.

\section{Materials and Methods}

Isolation of in Vivo Preimplantation Stages from Synchronized Donors. The estrus cycles of donor animals from the experimental herd of the Institute of Farm Animal Genetics (Holstein-Friesian cows, 3-5 years of age) were synchronized and all animals showing normal estrous underwent a gonadotrophin treatment with a total dose of $500 \mathrm{IU}$ porcine FSH (Pluset) to induce a mild superovulatory response as described previously $(44,45)$. The animals were kept under identical conditions and were fed according to milk yields. In vivo matured oocytes were aspirated from the preovulatory follicles of 9 animals on day 0 (= day of standing estrous) (46). For embryo collection, donor animals were inseminated with frozen/thawed semen samples from one HolsteinFriesian bull with proven fertility. Zygotes, 2-cell, 4-cell, and 8- to 16-cell stages were recovered by oviductal flushing using a minimally invasive endoscopic approach in 24 cows $1-4$ days after artificial insemination (AI). These oviductal stages were collected in prewarmed PBS containing 0.5\% BSA (45). Morulae 
and blastocysts were collected by uterine flushing at day 7 or 8 after $\mathrm{Al}$ (7 animals) using routine nonsurgical procedures. All embryos were carefully evaluated under a stereomicroscope and only morphologically intact embryos meeting established criteria (47) were used. Embryos were pooled in groups of 5, in the case blastocysts, or 10 embryos for the other stages, washed in PBS $+0.1 \%$ PVA, frozen in a minimum amount of medium in siliconized $0.6 \mathrm{~mL}$ cups and stored at $-80^{\circ} \mathrm{C}$. Typically, the time period between flushing and freezing was less than $30 \mathrm{~min}$. Animal treatments were conducted according to the German animal welfare guidelines.

In Vitro Maturation of Oocytes and in Vitro Production of Bovine Embryos. Due to the limited numbers of in vivo developed embryonic stages, in vitro produced counterparts were used for optimization of the Real Time PCR assay and to verify the expression patterns of several genes.

Bovine oocytes and embryos were produced in vitro from slaughterhouse ovaries as previously described $(48,49)$. For fertilization, semen was used from the same bull that had been used for Al of the donor animals (see above). Metaphase II oocytes (24 h maturation), zygotes, 2-cell, 4-cell, 8-cell, and blastocysts were harvested 19 h, 37-39 h, 39-40 h, day 3, day 5, and day 7/8 after fertilization (day $0=$ IVF). After washing three times in PBS containing $0.1 \%$ PVA the specimens were frozen at $-80{ }^{\circ} \mathrm{C}$ in a minimal volume $(5 \mu \mathrm{L})$ of PBS before RNA extraction.

Sampling Strategy and mRNA Isolation for Array Hybridization. Pools of 10 embryos were prepared for all stages except for the blastocyst stage when 5 were sufficient. Three pools each were prepared from in vivo metaphase II oocytes, zygotes, 8-cell embryos, morulae, and blastocysts, and two pools were prepared from 2-cell and 4-cell embryos, and independently processed for mRNA extraction. Poly(A)+ RNA was isolated with a Dynabeads mRNA Direct Kit (Invitrogen) according to the manufacturer's instruction with some modifications. Briefly, embryo pools were lysed in $40 \mu$ L of lysis-binding buffer (100 mM Tris- $\mathrm{HCl} \mathrm{pH}$ 8.0, $500 \mathrm{mM} \mathrm{LiCl,} 10 \mathrm{mM}$ EDTA, 1\% lithium dodecyl sulfate, $5 \mathrm{mM} \mathrm{DTT}$ ) and incubated at room temperature for $10 \mathrm{~min}$. Prewashed Dynabeads Oligo(dT) $25(5 \mu \mathrm{L})$ was added to the lysate followed by incubation for $5 \mathrm{~min}$ at room temperature on a shaker to allow binding of the poly $(A)+$ RNA to the beads. The beads were separated using a Dynal MPC-E-1 magnetic separator, washed once with washing buffer A (10 mM Tris- $\mathrm{HCl} \mathrm{pH} \mathrm{8.0,0.15}$ $\mathrm{mM} \mathrm{LiCl}, 1 \mathrm{mM}$ EDTA, $0.1 \%$ lithium dodecyl sulfate) and 2 times with washing buffer B (10 mM Tris-HCl, pH 8.0, $0.15 \mathrm{mM} \mathrm{LiCl}, 1 \mathrm{mM}$ EDTA). The poly(A)+ RNA's were eluted from the beads by incubation in $4 \mu \mathrm{L}$ sterile water at $65^{\circ} \mathrm{C}$ for $3 \mathrm{~min}$ and the mRNA's were used immediately as input for the first-strand CDNA synthesis using a T7 promoter-linked oligo(dT) primer following the standard protocol for the Affymetrix Two-Cycle cDNA Synthesis Kit (Affymetrix, Part. 900432). Protocols for the isolation and unbiased amplification of RNA from mammalian embryos have been published previously $(22,50)$. For this study, a system optimized for Affymetrix arrays was followed.

Amplification, Labeling, and Hybridization to DNA Microarrays. After secondstrand CDNA synthesis, biotin-labeled CRNA was prepared in an in vitro transcription reaction using the GeneChip IVT Labeling Kit (Affymetrix). Ten $\mu \mathrm{g}$ of fragmented cRNA was used for hybridization on the GenChip Bovine Genome Array (Affymetrix) overnight at $45^{\circ} \mathrm{C}$ according to the manufacturer's instructions.

DNA Microarray and Analysis of Array Data. The Affymetrix GeneChip Bovine Genome Array represents $>23,000$ transcripts, including assemblies from

1. Schultz RM (1993) Regulation of zygotic gene activation in the mouse. Bioessays 15:531-538.

2. Braude $P$, Bolton $V$, Moore $S$ (1988) Human gene expression first occurs between the four- and eight-cell stages of preimplantation development. Nature 332:459-461.

3. Telford NA, Watson AJ, Schultz GA (1990) Transition from maternal to embryonic control in early development: A comparison of several species. Mol Reprod Dev 26:90-100.

4. Misirlioglu M, et al. (2006) Dynamics of global transcriptome in bovine matured oocytes and preimplantation embryos. Proc Natl Acad Sci USA 103:18905-18910.

5. Kues WA, Niemann H (2004) The contribution of farm animals to human health. Trends Biotechnol 22:286-294.

6. Fadiel A, Anidi I, Eichenbaum KD (2005) Farm animal genomics and informatics: An update. Nucleic Acids Res 33:6308-6318.

7. Kues WA, Rath D, Niemann H (2008) Reproductive biotechnology in farm animals goes genomics. CAB Reviews: Perspect Agricult, Vet Sci, Nutr and Nat Resour 3, No. 036.

8. Ericson A, Källen B (2001) Congenital malformations in infants born after IVF: A population based study. Hum Reprod 16:504-509.

9. De Rycke M, Liebaerts I, Van Steirteghem A (2002) Epigenetic risks related to assisted reproductive technologies - Risk analysis and epigenetic inheritance. Hum Reprod 17:2487-2494.
19,000 UniGene clusters. The Bioconductor open source library (51) was used for initial processing of the raw files. CEL files were preprocessed using the GCRMA algorithm (which uses the GC content of probes in normalization with Robust MultiArray (RMA) normalization, GCRMA gives one value for each probe set instead of keeping probe level information) (Wu, et al., unpublished data) of the GCRMA package [v2.0.0], and detection calls, which indicate whether the transcript is detected $(P$, present), undetected $(A$, absent), or at the limit of detection ( $M$, marginal), were obtained using the mas5calls function $(52,53)$ of the Affymetrix platform. R version 2.4 .0 was used for implementing the packages of Bioconductor and also for plotting scatter plots. A matrix of Pearson's coefficient of correlation was created using R, which was in turn used to create the heat map, representing the correlation between the replicates. Filtering and compilations of data were carried out using MS Excel and MS Access. The TIGR-MEV (54; multiple experiment viewer from the Institute for Genomic Research, versatile microarray data analysis tool, incorporating sophisticated algorithms for clustering, visualization, classification, statistical analysis, and biological theme discovery) was used for significance tests and clustering. Before performing significance testing, genes having detection call " $A$ " in all of the stages were removed. One-way ANOVA was performed on this filtered list. To avoid the inclusion of false positives, $p$ values were calculated based on permutations, with a false discovery control, setting a cut off of proportion of false significant genes not exceeding 0.05 . With these stringent criteria, we got a significant list of 10,020 genes. Out of this set, after removing the genes which did not meet the requirement of a minimum cut off of 2 -fold between the maternal and embryonic stages, 9,263 genes were subjected to $\mathrm{K}$ means clustering, with a maximum of 50 iterations.

For pair-wise comparisons, a $t$ test with the Welch approximation, using a critical $P$ value of 0.05 was performed. Database for annotation, visualization and integrated discovery (DAVID; http://david.abcc.ncifcrf.gov) was used for pathway analyses.

To assess the overall similarity between the profiles of different replicates in the experiment, Pearson's correlation coefficient was calculated. Expression graphs of clusters were generated by the K-means clustering algorithm.

Pathway Analysis. Groups of genes associated with specific pathways, based on the Kyoto Encyclopedia of Genes and Genomes (KEGG), were analyzed together to assess pathway regulation during bovine embryo development.

Independent Verification of Array Data Using Real Time RT-PCR. Eight single oocytes/embryos and three pools of ten oocytes/embryos were thawed in $40 \mu \mathrm{L}$ of lysis-binding buffer, 1 pg of rabbit globin mRNA (BRL) was added to the solution as an internal standard. Poly(A)+RNA was prepared using a Dynabeads mRNA DIRECT KIT (Dynal) as described above and was eluted with $11 \mu \mathrm{L}$ sterile water. The eluted mRNA was immediately used as input for the reverse transcription reaction. Reverse transcription (RT) and Real-Time PCR were performed in 96-Well Optical Reaction Plates (Applied Biosystems). Primer sequences, fragment sizes, and references for sequences are summarized in Table S4.

ACKNOWLEDGMENTS. The authors gratefully acknowledge the expert assistance of Klaus-Gerd Hadeler in ovum pick up and oviductal and uterine embryo flushings, the excellent help by Karin Korsawe and Erika Lemme during embryo collection and grading, as well as the support from Martin Zenke and Bernd Denecke (RWTH Aachen) who performed chip hybridization. This study was supported by a grant from the Deutsche Forschungsgemeinschaft (DFG Forschergruppe 478) to H.N. and J.A..

10. Debaun MR, Niemitz EL, Feinberg AP (2003) Association of in vitro fertilization with Beckwith-Wiedemann syndrome and epigenetic alterations of LIT1 and H19. Am J Hum Genet 72:156-160.

11. Gicquel C, et al. (2003) In vitro fertilization may increase the risk of BeckwithWiedemann syndrome related to the abnormal imprinting of the KCNQ1OT gene. Am J Hum Genet 72:1338-1341.

12. Moll AC, et al. (2003) Incidence of retinoblastoma in children born after in vitro fertilisation. Lancet 361:309-310.

13. Powell K (2003) Seeds of doubt. Nature 422:656-658.

14. Halliday J, et al. (2004) Beckwith-Wiedemann syndrome and IVF: A case-control study. Am J Hum Genet 75:526-528.

15. Thompson EM, Legouy E, Renard JP (1998) Mouse embryos do not wait for the MBT: Chromatin and RNA polymerase remodeling in genome activation at the onset of development. Dev Genet 22:31-42.

16. Niemann H, Wrenzycki C (2000) Alterations of expression of developmentally important genes in preimplantation bovine embryos by in vitro culture conditions: Implications for subsequent development. Theriogenology 53:21-34.

17. Adjaye J, et al. (2004) Cross-species hybridisation of human and bovine orthologous genes on high density CDNA microarrays. BMC Genomics 5:83. 
18. Adjaye J, et al. (2007) Conserved molecular portraits of bovine and human blastocysts as a consequence of the transition from maternal to embryonic control of gene expression. Physiol Genomics 31:315-327.

19. Zhou D, Herrick DJ, Rosenbloom J, Chaqour B (2005) Cyr61 mediates the expression of VEGF, alpha-integrin, and alpha-actin genes through cytoskeletally based mechanotransduction mechanisms in bladder smooth muscle cells. J App/ Physio/ 98:2344-2354.

20. Nguyen TT, Sheppard AM, Kaye PL, Noakes PG (2007) IGF-I and insulin activate mitogen-activated protein kinase via the type 1 IGF receptor in mouse embryonic stem cells. Reproduction 134:41-49.

21. Bilodeau-Goeseels S, Schultz GA (1997) Changes in ribosomal ribonucleic acid content within in vitro-produced bovine embryos. Biol Reprod 56:1323-1329.

22. Brambrink T, et al. (2002) Application of CDNA arrays to monitor mRNA profiles in single preimplantation mouse embryos. Biotechniques 33:376-385.

23. Cabot RA, Hannink M, Prather RS (2002) CRM1-mediated nuclear export is present during porcine embryogenesis. Biol Reprod 67:814-819.

24. Campbell L, Hardwick KG (2003) Analysis of Bub3 spindle checkpoint function in Xenopus egg extracts. J Cell Sci 116:617-628.

25. Sudheer S, Adjaye J (2007) Functional genomics of human pre-implantation development. Brief Funct Genomic Proteomic 6:120-132.

26. Thélie $A$, et al. (2007) Differential regulation of abundance and deadenylation of maternal transcripts during bovine oocyte maturation in vitro and in vivo. BMC Dev Biol 7:125.

27. Duranthon $\mathrm{V}$, Watson $\mathrm{AJ}$, Lonergan $\mathrm{P}$ (2008) Preimplantation embryo programming: Transcription, epigenetics, and culture environment. Reproduction 135:141-150.

28. Fair T, Carter F, Park S, Evans AO, Lonergan P (2007) Global gene expression analysis during bovine oocyte in vitro maturation. Theriogenology 68:91-97.

29. Sharov AA, et al. (2003) Transcriptome analysis of mouse stem cells and early embryos. PLoS Biol 1:E74.

30. Hamatani T, et al. (2004) Global gene expression analysis identifies molecular pathways distinguishing blastocyst dormancy and activation. Proc Natl Acad Sci USA 101:10326-10331.

31. Wang QT, et al. (2004) A genome wide study of gene activity reveals developmental signalling pathways in the preimplantation mouse embryo. Dev Cell 6:133-144.

32. Su Al, et al. (2004) A gene atlas of the mouse and human protein-encoding transcriptomes. Proc Natl Acad Sci USA 101:6062-6067.

33. Niemann H, Carnwath JW, Kues W (2007) Application of DNA array technology to mammalian embryos. Theriogenology 68 (Suppl 1):S165-S177.

34. Barnes FL, Eyestone WH (1990) Early cleavage and the maternal to zygotic transition in bovine embryos. Theriogenology 33:141-152.

35. Marcucio RS, Hopwood RM, Ignotz GG, Currie WB (1995) Translation of zygoticallyderived mRNA in a cell cycle specific manner in 2-cell cattle embryos. $J$ Reprod Fert Abstr Series 15:abstract 46.

36. Hyttel P, Viuff D, Avery B, Laurincik J, Greve T (1996) Transcription and cell cycledependent development of intranuclear bodies and granules in two-cell bovine embryos. J Reprod Fert 108:263-270.
37. Memili E, Dominko T, First NL (1998) Onset of transcription in bovine oocytes and early embryos. Mol Reprod Dev 51:36-41.

38. Davis W, De Sousa PA, Schultz RM (1996) Transient expression of translation initiation factor elF-4c during the 2-cell stage of the preimplatation mouse embryo: Idenification by mRNA differential display and the role of DNA replication in zygotic gene activation. Dev Biol 174:190-201.

39. Kiefer JC (2007) Epigenetics in development. Dev Dynam 236:1144-1156.

40. Wrenzycki C, et al. (2005) Messenger RNA expression patterns in bovine embryos derived from in vitro procedures and their implications for development. Reprod Fert Develop 17:23-35.

41. King KK, Seidel GE, Jr, Elsden RJ (1985) Bovine embryo transfer pregnancies. I. Abortion rates and characteristics of calves. J Anim Sci 61:747-757.

42. King KK, Seidel GE, Jr, Elsden RJ (1985) Bovine embryo transfer pregnancies. II. Lengths of gestation. J Anim Sci 61:758-762.

43. Thibier M (2007) New records in the numbers of both in vivo-derived and in vitroproduced bovine embryos around the world in 2006. IETS-Newsletter 25(4):15-20.

44. Bungartz L, Niemann $\mathrm{H}$ (1994) Assessment of the presence of a dominant follicle and selection of dairy cows suitable for superovulation by single ultrasound examination. J Reprod Fert 101:583-591.

45. Besenfelder U, Havlicek V, Mösslacher G, Brem G (2000) Collection of tubal stage bovine embryos by means of endoscopy: A technique report. Theriogenology 55:837-845.

46. Bungartz L, Lucas-Hahn A, Rath D, Niemann H (1995) Collection of oocytes from cattle via follicular aspiration aided by ultrasound with or without gonadotropin pretreatment and in different reproductive stages. Theriogenology 43:667-675.

47. International Embryo Transfer Society (1998) Manual of the International Embryo Transfer Society (International Embryo Transfer Society, Savoy, IL), 3rd ed.

48. Eckert J, Niemann H (1996) Effects of platelet-derived growth factor (PDGF) on the in vitro production of bovine embryos in protein-free media. Theriogenology 46:307-320.

49. Schaetzlein S, et al. (2004) Telomere length is reset during early mammalian embryogenesis. Prod Natl Acad Sci USA 101:8034-8038.

50. Adjaye J, Bolton V, Monk M (1999) Developmental expression of specific genes detected in high-quality cDNA libraries from single human preimplantation embryos. Gene 237:373-383.

51. Gentleman RC, et al. (2004) Bioconductor: Open software development for computational biology and bioinformatics. Genome Biol 5:R80.

52. Liu HX, Cartegni L, Zhang MQ, Krainer AR (2001) A mechanism for exon skipping caused by nonsense or missense mutations in BRCA1 and other genes. Nat Genet 27:55-58.

53. Liu WM, et al. (2002) Analysis of high density expression microarrays with signed-rank cell algorithms. Bioinformatics 18:1593-1599.

54. Saeed Al, et al. (2003) TM4: A free open-source system for microarray data management and analysis. Biotechniques 34:374-378. 